BNL-94828-2010-IR

\title{
NSLS-II: Nonlinear Model Calibration for Synchrotrons
}

\author{
J. Bengtsson
}

October 2010

Photon Sciences Directorate

Brookhaven National Laboratory

\section{U.S. Department of Energy \\ DOE - Office of Science}

Notice: This manuscript has been authored by employees of Brookhaven Science Associates, LLC under Contract No. DE-AC02-98CH10886 with the U.S. Department of Energy. The publisher by accepting the manuscript for publication acknowledges that the United States Government retains a non-exclusive, paid-up, irrevocable, world-wide license to publish or reproduce the published form of this manuscript, or allow others to do so, for United States Government purposes.

This preprint is intended for publication in a journal or proceedings. Since changes may be made before publication, it may not be cited or reproduced without the author's permission. 


\section{DISCLAIMER}

This report was prepared as an account of work sponsored by an agency of the United States Government. Neither the United States Government nor any agency thereof, nor any of their employees, nor any of their contractors, subcontractors, or their employees, makes any warranty, express or implied, or assumes any legal liability or responsibility for the accuracy, completeness, or any third party's use or the results of such use of any information, apparatus, product, or process disclosed, or represents that its use would not infringe privately owned rights. Reference herein to any specific commercial product, process, or service by trade name, trademark, manufacturer, or otherwise, does not necessarily constitute or imply its endorsement, recommendation, or favoring by the United States Government or any agency thereof or its contractors or subcontractors. The views and opinions of authors expressed herein do not necessarily state or reflect those of the United States Government or any agency thereof. 


\section{Contents:}

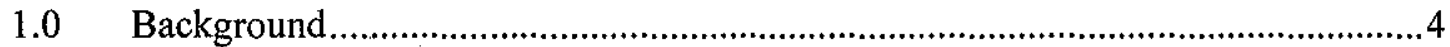

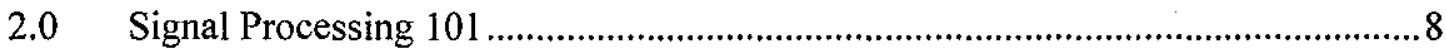

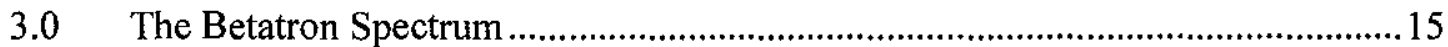

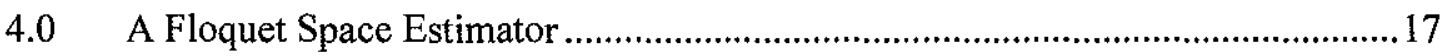

4.1 An Optimal Predictor-Corrector Algorithm: the Kalman Filter .......................................20

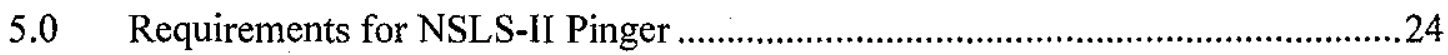

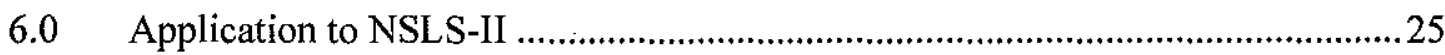

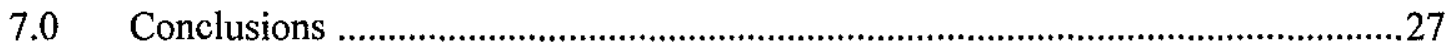

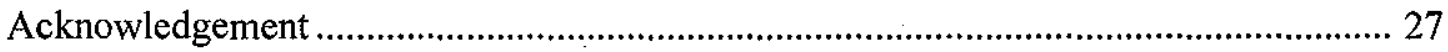

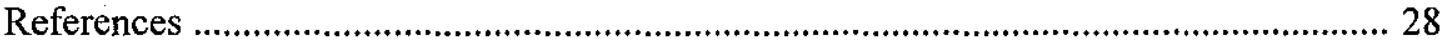




\subsection{Background}

This tech note is essentially a summary of a lecture we delivered to the Acc. Phys. Journal Club Apr, 2010 [1]. However, since the estimated accuracy of these methods has been naive and misleading in the field of particle accelerators, i.e., ignores the impact of noise, we will elaborate on this in some detail.

A prerequisite for a calibration of the nonlinear Hamiltonian is that the quadratic part has been understood, i.e., that the linear optics for the real accelerator has been calibrated. For synchrotron light source operations, this problem has been solved by the interactive LOCO technique/tool (Linear Optics from Closed Orbits) [2]. Before that, in the context of hadron accelerators, it has been done by signal processing of turn-by-turn BPM data [35].

A straightforward "reality check" of the nonlinear model can be done by:

1. An elementary test is to measure the on- and off momentum dynamic aperture. Traditionally, what's measured has not agreed well with what's been predicted; due to oversimplified models. Hence the poor estimates for Touschek life time. However, after the linear optics has been calibrated and corrected, and after introducing a realistic nonlinear model, the predictions and measurements actually do agree.

2. Another basic characteristic, and key design metric, is the tune footprint [6]. It can be measured by (elementary) signal processing of turn-by-turn BPM data for different amplitudes. It has become fashionable to use ${ }^{1}$

$$
\operatorname{Max}\{|X(v)|\}=\operatorname{Max}\left\{\left|\left\langle w_{k} x_{k}, e^{i 2 \pi k v}\right\rangle_{N}\right|\right\} \equiv \operatorname{Max}\left\{\left|\sum_{k=0}^{N-1} w_{k} x_{k} e^{-i 2 \pi k v}\right|\right\}
$$

i.e., to solve numerically for a Hann window

$$
w_{k}=\sin ^{2}\left(\frac{\pi k}{N}\right), \quad 0 \leq k \leq N-1
$$

A more direct, i.e., analytic approach, is to first compute the Discrete Fourier Transform (DFT) and then do a nonlinear interpolation in the spectrum. In fact, this is how we calibrated- and improved the nonlinear dynamics for LEAR (antiprotons), CERN in the mid-80s [3]. In particular, our accuracy was $\sim 1 \times 10^{-5}$ for 1024 turns (to calibrate the optics) and $\sim 1 \times 10^{-4}$ for 256 turns (to correct the linear coupling), see Figs. 1-2. The measured amplitude dependent tune shift before- and after nonlinear corrections

1. Aka NAFF (Numerical Analysis of Fundamental Frequency); with a component wise spectrum deconvolution by Gramm-Schmidt orthogonalization [7]. 
are shown in Fig. $3^{1}$. Later, at SPS, CERN in the mid-90s, they achieved e.g. $\sim 1 \times 10^{-4}$ for 128 turns [8].

3. A more detailed footprint of the nonlinear dynamics is the betatron spectrum, see Fig. 4. The frequencies, amplitudes, and phases are obtained by basic signal processing, see section 2.0 .

For more advanced work, one may attempt to correct the observed resonances [9-10]. Actually, this is how we corrected linear coupling in LEAR ${ }^{2}$, compare Figs. 4-5; a prerequisite for improving the nonlinear dynamics.

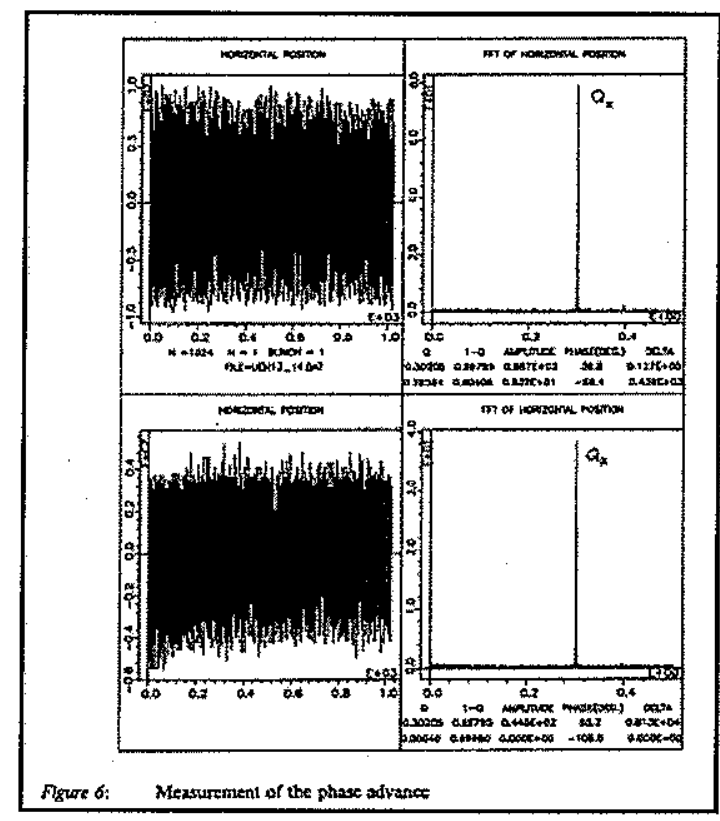

FIGURE 1. Horizontal Betatron Spectrum for LEAR $(N=1048)$.

1. To obtain good agreement between the model and measured values, sextupole like kinematic (coriolis) terms originating from the curved trajectories in the dipoles had to be included; due to the small circumference of LEAR $(80 \mathrm{~m})$ [3].

2. First by introducing a $3.2 \mathrm{mrad}$ roll (with a big crescent wrench) to one of the normal quadrupoles at a suitable location (determined by the measured phase); because LEAR did not have any skew quadrupole correctors. And, second, by moving the sextupoles onto the vertical orbit; since feed-down from ditto (no vertical orbit correctors) turned out to be the main source [3]. 


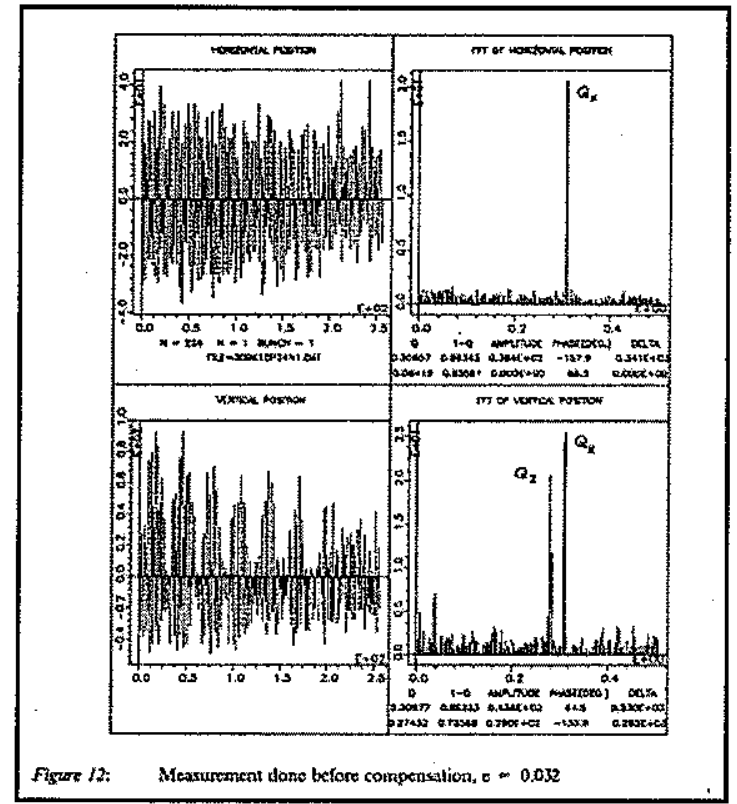

FIGURE 2. Horizontal- and Vertical Betatron Spectrum for $\operatorname{LEAR}(N=256)$.

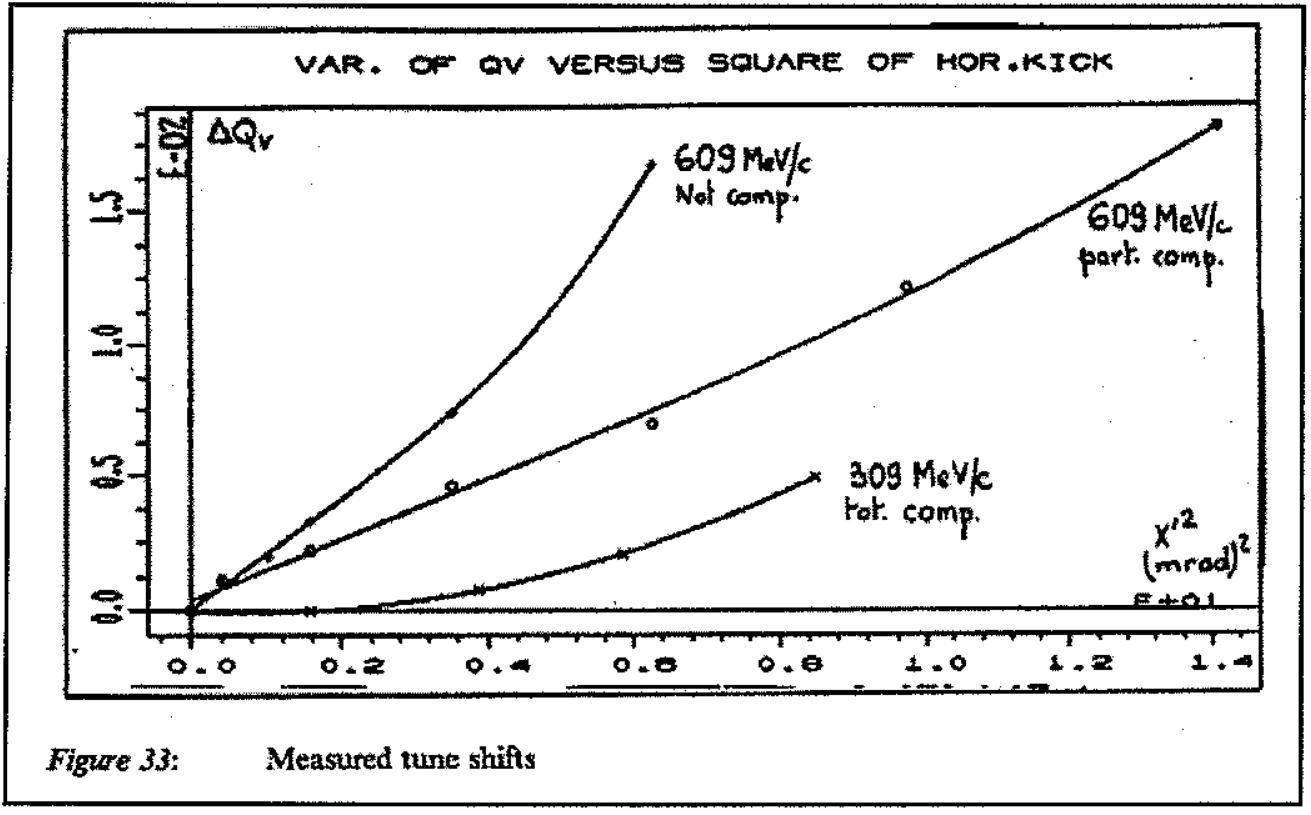

FIGURE 3. Measured Amplitude Dependent Tune Shift in LEAR Before- and After Nonlinear Corrections. 


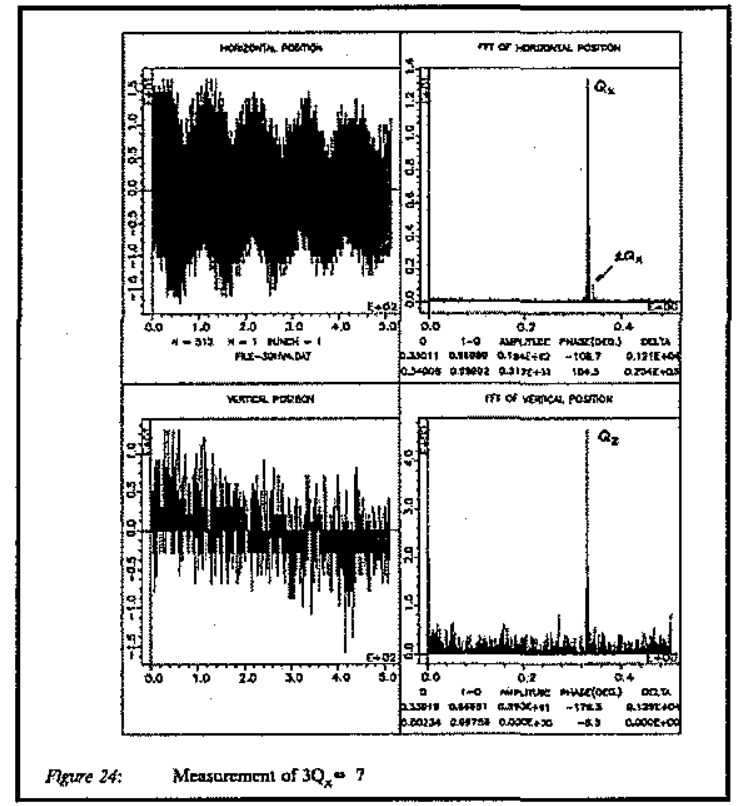

FIGURE 4. Measurement of the Extraction Resonance $3 v_{x}=7$ in LEAR.

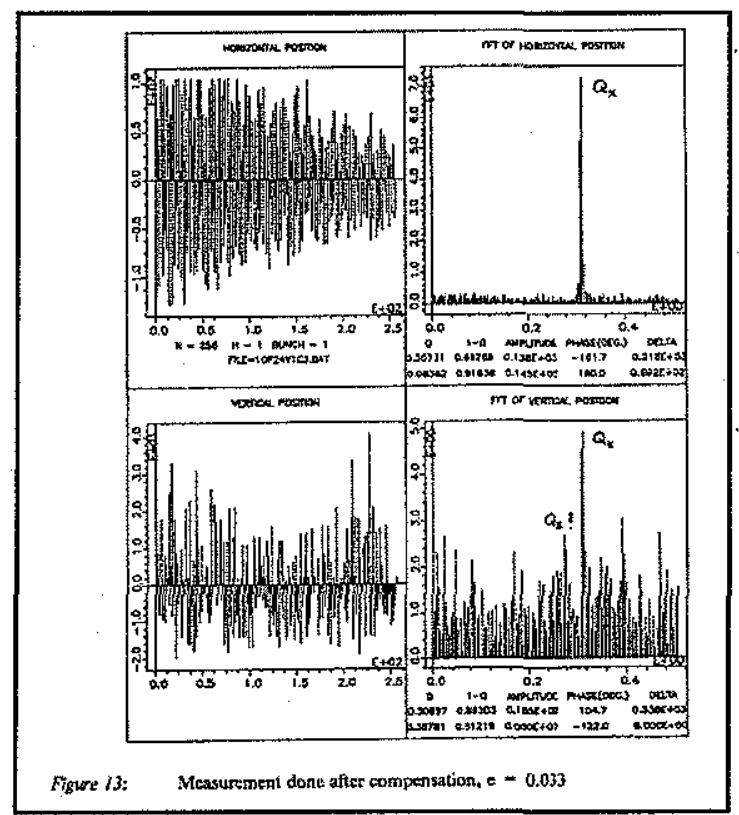

FIGURE 5. Horizontal- and Vertical Betatron Spectrum for LEAR After Correction of Linear Coupling. 


\subsection{Signal Processing 101}

Over the last decade(s), there has been a rediscovery of basic signal processing algorithms in the field of accelerators. However, the estimated accuracy in this field has been naive and misleading; because the impact of noise has been ignored. For a recent exception, i.e., a straightforward analysis of the effect of noise by simulation, see ref. [11]. Needless to say, these techniques have been well known in: telecommunication (taking noise into account) since the 40s [12-13], oceanography since the 60s [14], and celestial mechanics since the early 80 s [15]. In fact, the FFT algorithm, which is credited to Cooley \& Tukey [16], who published to put it into the local domain [17], had already been developed for Xray crystallography in the 40s [18], and by Gauss ca 1805 [19]; to interpolate the trajectories for the asteroids Pallas and Juno [20]. Today there is even a standard (IEEE Std. 10571994) for 3- and 4-parameter sine wave fit algorithms [21].

To recap, the Fourier transform is defined by

$$
f(t)=\frac{1}{2 \pi} \int_{-\infty}^{\infty} F(\omega) e^{i \omega t} d \omega
$$

where

$$
F(\omega)=\int_{-\infty}^{\infty} f(t) e^{-i \omega t} d t
$$

In other words, introduce the basis $e^{i \omega t}$ and the scalar product

$$
\langle f(t), g(t)\rangle=\int_{-\infty}^{\infty} f(t) \bar{g}(t) d t
$$

i.e., $F(\omega)=\left\langle f(t), e^{i \omega t}\right\rangle$. It follows that 


$$
\begin{aligned}
e^{i \omega_{0} t} f(t) & \rightarrow F\left(\omega-\omega_{0}\right), \\
1 & \rightarrow 2 \pi \delta(\omega), \\
\delta(t) & \rightarrow 1, \\
e^{-|\alpha| t}(t) & \rightarrow \frac{2 \alpha}{\alpha^{2}+\omega^{2}}, \\
\operatorname{rect}\left(\frac{t}{T}\right) & \rightarrow T \operatorname{sinc}\left(\frac{\omega T}{2}\right), \\
\cos \left(\frac{\pi t}{T}\right) \operatorname{rect}\left(\frac{t}{T}\right) & \rightarrow \frac{\pi T}{2} \frac{\sin (\omega T / 2-\pi / 2)}{(\omega T / 2)^{2}-(\pi / 2)^{2}}, \\
\cos ^{2}\left(\frac{\pi t}{T}\right) \operatorname{rect}\left(\frac{t}{T}\right) & \rightarrow-\frac{T}{2} \frac{\pi^{2}}{(\omega T / 2)^{2}-\pi^{2}} \operatorname{sinc}\left(\frac{\omega T}{2}\right)
\end{aligned}
$$

where

$$
\begin{aligned}
\operatorname{rect}\left(\frac{t}{T}\right) & \equiv \Theta(t+T / 2)-\Theta(t-T / 2), \\
\operatorname{sinc}(\omega T) & \equiv \frac{\sin (\omega T)}{\omega T}
\end{aligned}
$$

For a sampled system, with sample rate $f_{s}=1 / \Delta t$ at discrete times $k \Delta t$ over some interval $T=N \Delta t$, one may introduce the normalized frequency

$$
v_{s}=\frac{f}{f_{s}}
$$

so that

$$
\omega t=2 \pi v_{s} k
$$

and evaluate the transform for the discrete frequencies

$$
v_{s}=\frac{n}{N} .
$$


one obtains the Discrete Fourier Transform (DFT) ${ }^{1}$ of a N-periodic discrete-time sequence $x_{k}, k=0,1, \ldots, N-1^{2}$

$$
x_{k}=\sum_{k=0}^{N-1} X_{n} e^{i 2 \pi k n / N}, \quad k=0,1, \ldots, N-1
$$

where

$$
X_{n}=\frac{1}{N} \sum_{k=0}^{N-1} x_{k} e^{-i 2 \pi k n / N}, \quad n=0,1, \ldots, N-1
$$

In other words, introduce the (discrete) orthogonal basis $e^{i 2 \pi k n / N}$ and the (discrete) scalar product

$$
\left\langle x_{i}, y_{j}\right\rangle_{N}=\frac{1}{N} \sum_{k=0}^{N-1} x_{k} \bar{y}_{k}
$$

The "coordinates" $X_{n}$ for the $N$ samples $x_{k}, k=0,1, \ldots, N-1$ of any complex N-periodic function is

$$
X_{n}=\left\langle x_{k}, e^{i 2 \pi k n / N}\right\rangle_{N}
$$

Due to the finite time window, a discrete peak has side lobes in the spectrum. These can be reduced by a suitable choice of window function $w_{k}, k=0,1, \ldots, N-1$. The Fourier transform for the typical choices are

$$
\begin{array}{rlrl}
\text { Rectangular: } & e^{i 2 \pi k v_{0}} \operatorname{rect}\left(\frac{k}{N}\right) & \rightarrow \operatorname{sinc}\left(\pi\left(n-N v_{0}\right)\right), \\
\text { Sine: } & e^{i 2 \pi k v_{0}} \sin \left(\pi \frac{k}{N}\right) \rightarrow \frac{1}{2 \pi} \frac{\sin \left(\pi\left(n-N v_{0}-1 / 2\right)\right)}{\left(n-N v_{0}\right)^{2}-(1 / 2)^{2}}, \\
\text { Hann: } & e^{i 2 \pi k v_{0}} \sin ^{2}\left(\pi \frac{k}{N}\right) \rightarrow-\frac{1}{2} \frac{1}{\left(n-N v_{0}\right)^{2}-1} \operatorname{sinc}\left(\pi\left(n-N v_{0}\right)\right)
\end{array}
$$

1. A straightforward implementation of DFT leads to an $O\left(N^{2}\right)$ process. Fast Fourier Transform (FFT) is an algorithm to compute it as $O\left(N \log _{2}(N)\right)$ when $N=2^{n}$ for some $n$. In fact, FFT is what made digital filters, etc. practical; e.g. digital music.

2. The interval is now $[0, T]$ instead of $[-T / 2, T / 2]$. 
for $n / N \approx v_{0}$. In other words, the amplitude of the side lobes are suppressed on behalf of the width of the main peak. For completeness, the exact expressions are obtained from Eq. (12). For e.g. a rectangular window it is

$$
\frac{1}{N} \frac{1-e^{-i 2 \pi\left(n-N v_{0}\right)}}{1-e^{-i 2 \pi\left(n / N-v_{0}\right)}}=\frac{1}{N} \frac{\sin \left(\pi\left(n-N v_{0}\right)\right)}{\sin \left(\pi\left(n / N-v_{0}\right)\right)}=\frac{\sin \left(\pi\left(n-N v_{0}\right)\right)}{\pi\left(n-N v_{0}\right)}+\ldots
$$

Two-step (nonlinear) interpolation formula for a signal with one frequency are

$$
\begin{array}{rlrl}
\text { Rectangular: } & & v=\frac{1}{N}\left(n-1+\frac{1}{1+A_{n-1} / A_{n}}\right), \\
\text { Sine: } & v=\frac{1}{N}\left(n-\frac{3}{2}+\frac{2}{1+A_{n-1} / A_{n}}\right), \\
\text { Hann: } & v=\frac{1}{N}\left(n-2+\frac{3}{1+A_{n-1} / A_{n}}\right)
\end{array}
$$

where $\left(A_{n}=\left|X_{n}\right|\right), n-1 \leq N v \leq n^{1}$. The interpolation formula for the Hann window is an analytic solution which, in the ideal case of only one peak, is equivalent to the numerical solution of

$$
\operatorname{Max}\left\{\left|\left\langle w_{k} x_{k}, e^{i 2 \pi k v}\right\rangle_{N}\right|\right\}
$$

by NAFF. Neither of these estimates is exact even in the ideal case though, i.e., for a signal with one frequency and no noise, due to aliasing ${ }^{2}$. However, due to the side lobe suppression for the latter two windows, $v$ just need to be a few bins away from 0 and 0.5 .

The accuracy for a DFT without interpolation is $\sim 1 / N$. And from Eqs. (15) it is clear that the reduction of the side lobes are

$$
\sim 1 / N^{\alpha}, \alpha=1,2,3
$$

respectively. So the "accuracy" (without noise) is

$$
\sim 1 / N^{\alpha}, \alpha=2,3,4
$$

1. For best Signal-to-Noise-Ratio, i.e., $n$ is arbitrary.

2. Missed by Laskar [7]. 
respectively ${ }^{1}$. However, this analysis is academic, because the impact of noise is $\sim 1 / N^{3 / 2}$; for any of the methods. In particular, differentiating Eqs. (17) gives

$$
d v=\frac{1}{N\left(1+A_{n-1} / A_{n}\right)^{2}}\left(\frac{d A_{n-1}}{A_{n}}-\frac{A_{n-1}}{A_{n}^{2}} d A_{n}\right)
$$

and it follows that

$$
\left\langle d v^{2}\right\rangle \leq \frac{1}{N^{2}}\left\langle\left(\frac{d A_{n-1}}{A_{n-1}}-\frac{d A_{n}}{A_{n}}\right)^{2}\right\rangle \leq \frac{2}{N^{2}}\left\langle\left(\frac{d A_{n}}{A_{n}}\right)^{2}\right\rangle=\frac{1}{\mathrm{SNR}^{2}} \frac{2}{N^{2}} \sim \frac{2}{N^{3}}
$$

where the Signal-to-Noise-Ratio (SNR) is the ratio between rms signal to rms noise

$$
\frac{1}{\mathrm{SNR}^{2}}=\operatorname{Var}\left\{\frac{d A_{n}}{A_{n}}\right\} \sim \frac{1}{N}
$$

Regardless, it is substantial improvement. For e.g. $N=256$ and $5 \%$ noise we obtain $\delta v \sim 1 \times 10^{-5}$. In fact, the interpolation technique developed by E. Asséo ${ }^{2}$ [23] is precisely what made our, at the time, novel method to calibrate- and improve the nonlinear dynamics for LEAR, CERN (antiprotons) in the mid-80 s practical; and a success ${ }^{3}$.

Alternatively, in the time domain a least-square formulation is given by

$$
\chi^{2}(\bar{a})=\sum_{k=1}^{i v}\left(\frac{x_{k}-y_{k}(\bar{a})}{\sigma_{k}}\right)^{2}=\sum_{k=1}^{i v}\left(\frac{x_{k}-A \sin (2 \pi k v+\varphi)}{\sigma_{k}}\right)^{2}
$$

with the parameters $\bar{a}=[v, A, \varphi]$. Note that $\partial \chi^{2}(v, A, \varphi) / \partial v=0$ for the solution of the least-square problem, so the error is given by $\partial^{2} \chi^{2}(v, A, \varphi) / \partial v^{2}$. In fact, the variance of the estimated parameters with a confidence level CL for a general least-square fit is [24]

$$
\left(\delta a_{i}\right)^{2}=\Delta \chi_{\mathrm{CL}}^{2} \cdot C
$$

1. The same result was obtained from the analysis in ref. [22], i.e., for the suppression of other frequencies; not noise.

2. An electrical engineer, that designed the electronics for the turn-by-turn BPM data acquisition system at LEAR, with an in-depth understanding of noise, how to e.g. use a programmable HP-calculator to simulate/test/evaluate/improve a design, method, etc.

3. For example, the first anti-hydrogen atom was produced by one of the experiments at LEAR in 1995. 
where $\Delta \chi_{\mathrm{CL}}^{2}$ is the Confidence Level, $C$ the covariance matrix

$$
C=[\alpha]^{-1}
$$

and $[\alpha]$ the curvature matrix

$$
\alpha_{i j}=\sum_{k=1}^{N} \frac{1}{\sigma_{k}^{2}} \frac{\partial y_{k}(\bar{a})}{\partial \alpha_{i}} \frac{\partial y_{k}(\bar{a})}{\partial \alpha_{j}}
$$

In particular, for a 1-parameter fit of $v$ we obtain

$$
\begin{aligned}
\alpha_{11} & =\sum_{k=1}^{N} \frac{(2 \pi k A)^{2}}{\sigma_{k}^{2}} \cos ^{2}(2 \pi k v+\varphi) \rightarrow 2 \pi^{2} \mathrm{SNR}^{2} \sum_{k=1}^{N} k^{2} \\
& =\mathrm{SNR}^{2} \cdot \frac{\pi^{2} N(N+1)(2 N+1)}{3} \geq \frac{2 \pi^{2}}{3} \mathrm{SNR}^{2} N^{3}
\end{aligned}
$$

for large $N$, where we have introduced $\mathrm{SNR} \equiv A / \sigma$ and assumed that $\sigma_{k}=\sigma$. It follows that

$$
(\delta v)^{2} \leq \frac{3}{2 \pi^{2}} \frac{1}{\mathrm{SNR}^{2}} \frac{1}{N^{3}} \sim \frac{1}{N^{3}}
$$

since the SNR for the signal in the time domain is fixed.

Bottom line, the information content from a DFT and interpolation in the frequency space, or a least-square fit in the temporal space are equivalent (in the sense of information content available from either space). In particular, the impact of noise is the same; something that we stated on the last slide in our April lecture [1].

Clearly, the accuracy can be improved even further by using all the BPMs. For example, the least-square can be generalized to

$$
\chi^{2}(\bar{a})=\sum_{i=1} \sum_{k=1}^{j v_{\mathrm{BPM}} v_{\mathrm{tum}}}\left(\frac{x_{j k}-y_{j k}(\bar{a})}{\sigma_{j k}}\right)^{2}=\sum_{j=1} \sum_{k=1}^{j v_{\mathrm{BPM}} v_{\mathrm{tum}}}\left(\frac{x_{j k}-A_{j} \sin \left(2 \pi k v+\varphi_{j}\right)}{\sigma_{j k}}\right)^{2}
$$

with $\bar{a}=\left[v, A_{j}, \varphi_{j}\right], j=1,2, \ldots, N_{\mathrm{BPM}}$.

Alternatively, the optics at each BPM can be obtained from individual FFTs; as we demonstrated in ref. [3]. However, a more elegant approach, is to consider $x_{j k}$ as a matrix and compute the Singular Value Decomposition (SVD) [24] 


$$
X=U \operatorname{diag}\left\{w_{k}\right\} V^{T}, \quad U^{T} U=I, \quad V^{T} V=V V^{T}=I
$$

Note that

$$
X X^{T}=U \operatorname{diag}\left\{w_{k}^{2}\right\} U^{T}, \quad X^{T} X=V \operatorname{diag}\left\{w_{k}^{2}\right\} V^{T}
$$

Unfortunately, this has been advertised" as "Model Independent Analysis" (MIA) $\Rightarrow$ pure nonsense. Clearly, if the motion was not assumed to be quasi periodic and deterministic, the pick-up electrodes not assumed to respond to the center-of-charge of the bunches, the response of the diagnostics not assumed to be linear, etc., either approach would be meaningless. Just another symptom of a reductionist ${ }^{2}$ point of view; or inflating the application of a known numerical technique to a "novel method". In fact, the approach is more generally known as Principal Component Analysis (PCA); a century old method in statistical analysis [25-26]. Roughly, a variance based, orthogonal decomposition of large data sets to a small set of significant components. On the contrary, for the PCA (statistical) results to be meaningful (physics), for e.g. a calibration of the (linear) optics model, they must be (mathematically) linked to the beam dynamics model.

In 1 D.O.F. the local amplitudes and phases can be estimated from [27]

$$
A_{j}^{*}=\left(w_{1} v_{j 1}\right)^{2}+\left(w_{2} v_{j 2}\right)^{2}, \quad \varphi_{j}^{*}=-\operatorname{atan}\left(\frac{w_{2} v_{j 2}}{w_{1} v_{j 1}}\right)
$$

and the tune is obtained by a FFT of $u_{k 1}$ or $u_{k 2}$, where

$$
U \equiv\left[u_{j k}\right], \quad V \equiv\left[v_{j k}\right]
$$

Again, we conjecture that the noise suppression for tune measurements in either case (least-square or SVD) is

$$
\sim \frac{1}{\sqrt{N_{\mathrm{BPM}} \cdot N_{\text {turn }}}}
$$

whereas for optics measurements (by least-square, individual FFTs, or SVD) we expect

$$
\sim \frac{1}{\sqrt{N_{\text {turn }}}}
$$

1. Contributing to the confusions regarding known signal processing methods in this field, rather than a comparative, quantitative analysis (including the effect of noise); aka an objective/experimentalist approach.

2. A component, rather than system, based view/interpretation (e.g. ignoring the effect of noise) of the world of physical phenomena. 
which may be demonstrated by simulations. For better spectral decomposition of the harmonics in 2- or 3 D.O.F., one may use Independent Component Analysis (ICA) [28].

\subsection{The Betatron Spectrum}

A more detailed footprint is the betatron spectrum. A simulation for NSLS-II main ring with $\bar{A}=[10,2] \mathrm{mm}=>\bar{v}=[33.117,16.195]$ is shown in Fig. 6 . The linear coupling$v_{x} \pm v_{y}$ and leading order sextupolar resonances $v_{x} \pm 2 v_{y}, 3 v_{x}$, generates

$v_{y}(0.12), 2 v_{x, y}(0.23,0.39)$ and $v_{x}(0.20), v_{x} \pm v_{y}(0.31,0.08)$ sidebands in the horizontal- and vertical betatron spectrum, respectively [3].

To improve the resolution, one may instead Fourier analyze the linear action variables [29], which are directly related to the resonance terms in the Hamiltonian, see Fig. 7. The linear coupling- $v_{x} \pm v_{y}(0.31,0.078)$, first order sextupolar-

$v_{x}(0.11), v_{x} \pm 2 v_{y}(0.49,0.27), 3 v_{x}(0.35)$, and second order resonances

$2 v_{x, y}(0.23,0.39), 2 v_{x} \pm 2 v_{y}(0.38,0.16), 4 v_{x, y}(0.47,0.22)$ are now transparent.

In particular, a driving term $h_{I}[29]$

$$
h_{\bar{I}}+h_{\bar{I}}^{*}=2\left|h_{\bar{I}}\right| \cos \left(\phi_{\bar{I}}\right), \quad \bar{I} \equiv\left[i_{1}, i_{2}, i_{3}, i_{4}, i_{5}\right]
$$

perturbs the initial linear action $\bar{J}_{0}$ by

$$
\begin{aligned}
& J_{x k}=J_{x 0}+m_{x} g_{\bar{I} k}+O\left(h_{I}^{2}\right), \\
& J_{y k}=J_{y 0}+m_{y} g_{\bar{I} k}+O\left(h_{\bar{I}}^{2}\right)
\end{aligned}
$$

where

$$
\begin{aligned}
& g_{I k}=\frac{\left|h_{\bar{I}}\right|\left(2 J_{x 0}\right)^{m_{x} / 2}\left(2 J_{y 0}\right)^{m_{y} / 2} \delta^{i_{5}}}{\sin \left(\pi\left[n_{x} v_{x}+n_{y} v_{y}\right]\right)} \cos \left(\phi_{\bar{I} 0}+k 2 \pi\left[n_{x} v_{x}+n_{y} v_{y}\right]\right), \\
& \phi_{\bar{I} 0}=\phi_{\bar{I}}+n_{x}\left(\phi_{x 0}-\pi v_{x}\right)+n_{y}\left(\phi_{y 0}-\pi v_{y}\right)
\end{aligned}
$$

and

$$
m_{x}=i_{1}+i_{2}, \quad m_{y}=i_{3}+i_{4}, \quad n_{x}=i_{1}-i_{2}, \quad n_{y}=i_{3}-i_{4}
$$

i.e., generates to leading order a peak at

$$
v=n_{x} v_{x}+n_{y} v_{y}
$$




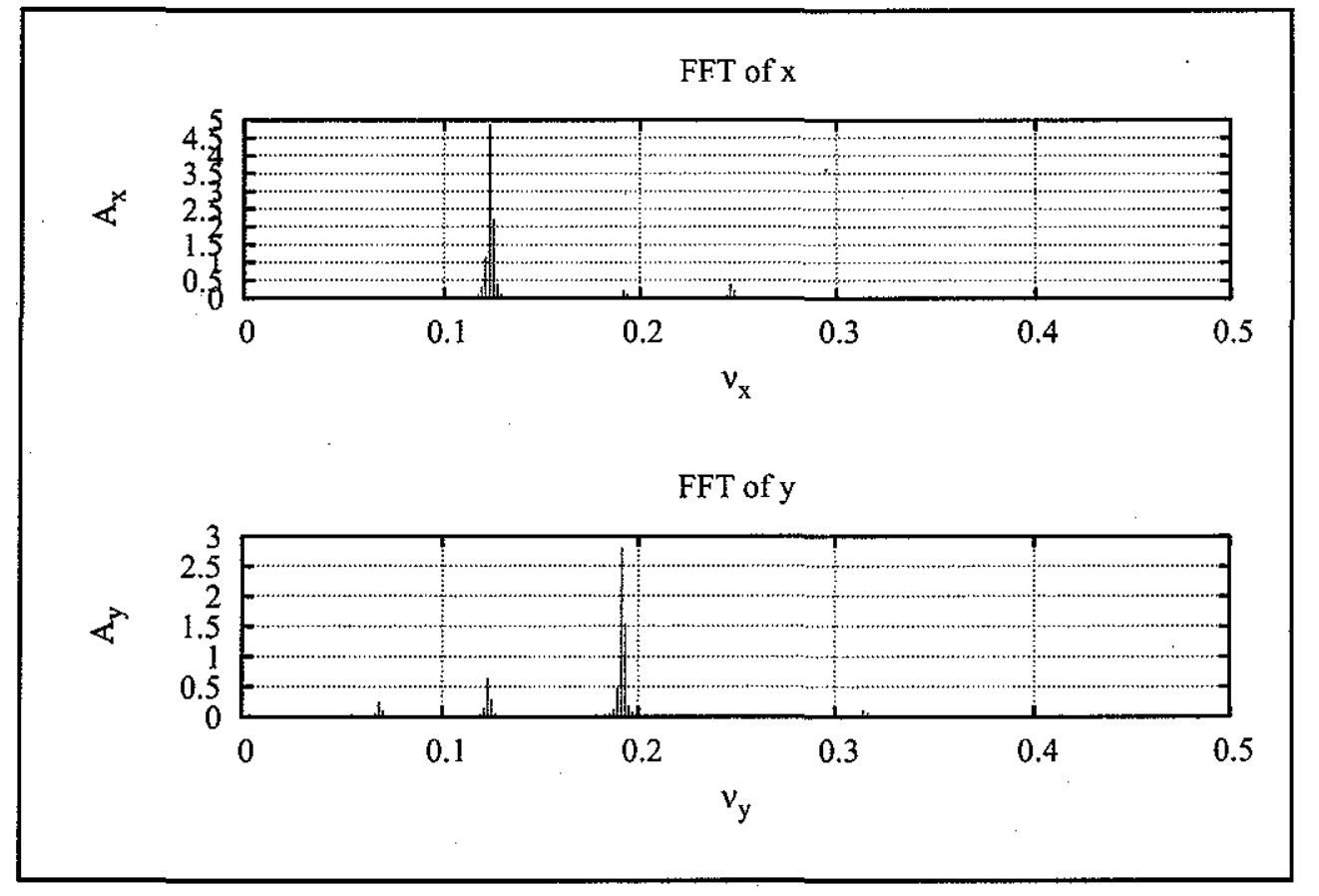

FIGURE 6. Betatron Spectrum, $v_{x, y}=[33.12,16.19]$

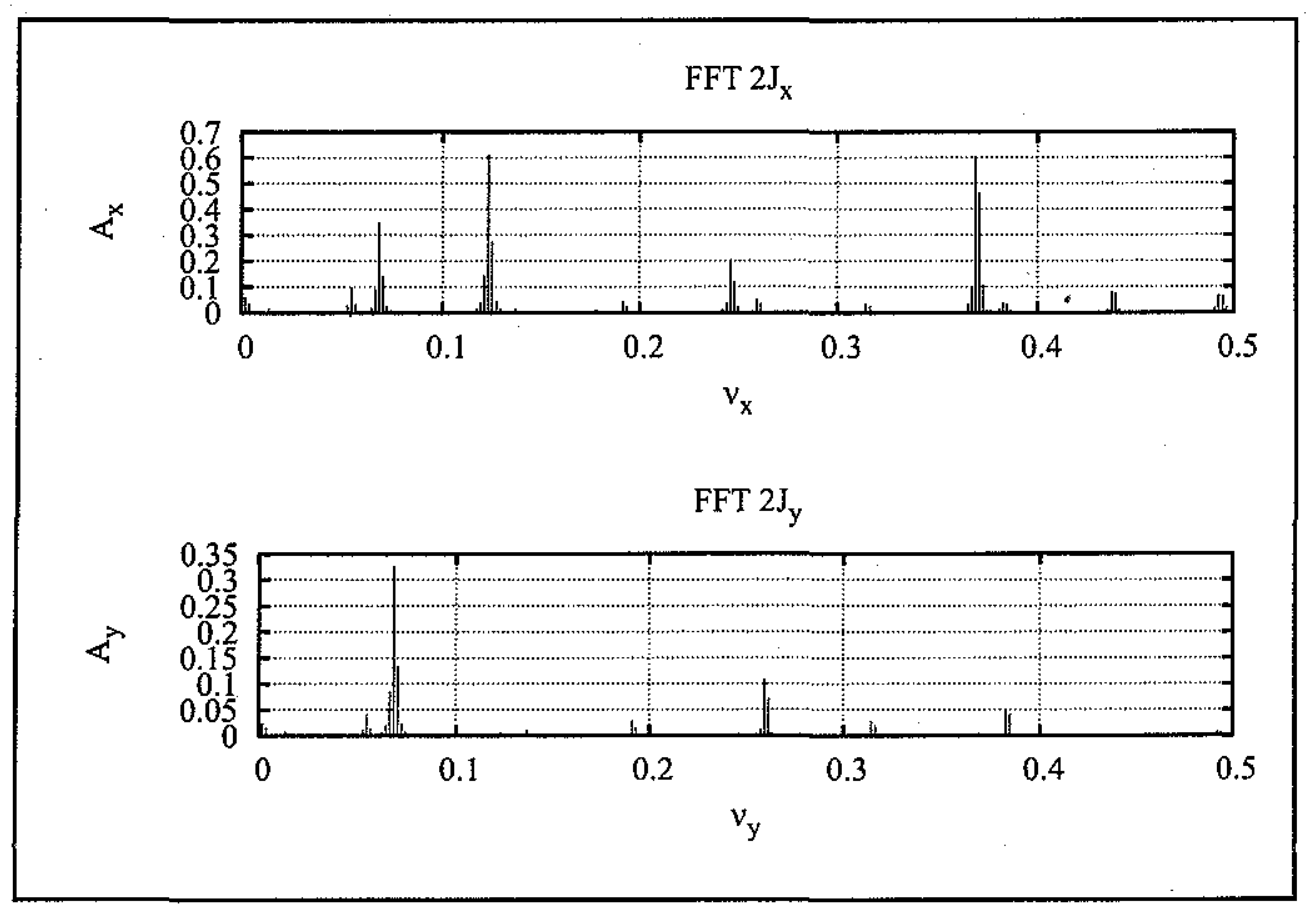

FIGURE 7. Spectrum of $2 J_{x, y}$. 


\subsection{A Floquet Space Estimator}

Since Hill's equation (linear) is observable, the state-space, i.e., $\left[x, x^{\prime}, y, y^{\prime}\right]$, can be reconstructed with turn-by-turn data from one BPM [30]. However, to analyze the variation of the linear action variables due to the nonlinear dynamics, we need a pair of BPMs ${ }^{1}$ separated by only linear elements (i.e., drifts, dipoles, and quadrupoles).

The transport matrix. between the two BPMs is ${ }^{2}$

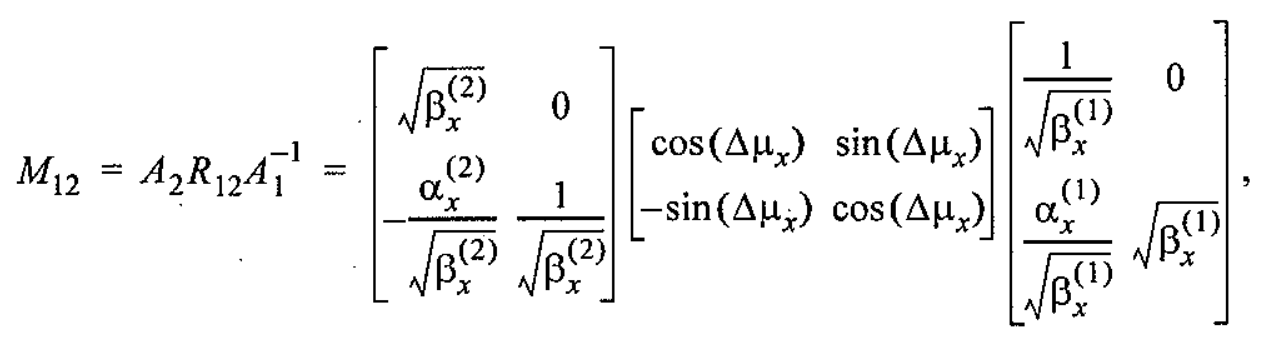

with the (linear) center-of-charge motion at the first BPM given by

$$
\begin{aligned}
& x_{k}^{(1)}=\sqrt{2 J_{x} \beta_{x}^{(1)}} \cos \left(k 2 \pi v_{x}+\phi_{x}^{(1)}\right), \\
& p_{x k}^{(1)}=-\sqrt{\frac{2 J_{x}}{\beta_{x}^{(1)}} \alpha_{x}^{(1)} \sin \left(k 2 \pi v_{x}+\phi_{x}^{(1)}\right)+\sqrt{\frac{2 J_{x}}{\beta_{x}^{(1)}}} \cos \left(k 2 \pi v_{x}+\phi_{x}^{(1)}\right)}
\end{aligned}
$$

and similarly at the second BPM. From the ( $N$-turn) averages

$$
\begin{aligned}
\left\langle\left(x_{k}^{(1)}\right)^{2}\right\rangle & =J_{x} \beta_{x}^{(1)}, \quad\left\langle\left(x_{k}^{(2)}\right)^{2}\right\rangle=J_{x} \beta_{x}^{(2)}, \\
\left\langle x_{k}^{(1)} x_{k}^{(2)}\right\rangle & =J_{x} \sqrt{\beta_{x}^{(1)} \beta_{x}^{(2)}} \cos \left(\Delta \mu_{x}\right)
\end{aligned}
$$

we obtain an estimate of the ratio of the beta functions and phase advance between the two BPMs

$$
\begin{aligned}
\frac{\beta_{x}^{(1)^{*}}}{\beta_{x}^{(2)}} & =\frac{\left\langle\left(x_{k}^{(1)}\right)^{2}\right\rangle}{\left\langle\left(x_{k}^{(2)}\right)^{2}\right\rangle}, \\
\left(\Delta \mu_{x}\right)^{*} & =\operatorname{acos}\left(\frac{\left\langle x_{k}^{(1)} x_{k}^{(2)}\right\rangle}{\sqrt{\left\langle\left(x_{k}^{(1)}\right)^{2}\right\rangle\left\langle\left(x_{k}^{(2)}\right)^{2}\right\rangle}}\right),
\end{aligned}
$$

1. Preferable in a non dispersive region; to avoid a strong synchrotron sideband.

2. Assuming mid-plane symmetry, since the (linear) optics has been calibrated/corrected. 
whereas $\sqrt{\beta_{x}^{(1)}}$ is obtained from the linear optics; which we have assumed is known.

In Floquet space we have

$$
\begin{aligned}
& \tilde{x}_{k}^{(2)}=\tilde{x}_{k}^{(1)} \cos \left(\Delta \mu_{x}\right)+\tilde{p}_{x k}^{(1)} \sin \left(\Delta \mu_{x}\right), \\
& \tilde{p}_{x k}^{(2)}=-\tilde{x}_{k}^{(1)} \sin \left(\Delta \mu_{x}\right)+\tilde{p}_{x k}^{(1)} \cos \left(\Delta \mu_{x}\right)
\end{aligned}
$$

which gives

$$
\tilde{p}_{x k}^{(1)}=\frac{\tilde{x}_{k}^{(2)}-\tilde{x}_{k}^{(1)} \cos \left(\Delta \mu_{x}\right)}{\sin \left(\Delta \mu_{x}\right)}=\frac{\sqrt{\beta_{x}^{(1)} / \beta_{x}^{(2)}} x_{k}^{(2)}-x_{k}^{(1)} \cos \left(\Delta \mu_{x}\right)}{\sqrt{\beta_{x}^{(1)}} \sin \left(\Delta \mu_{x}\right)}
$$

where

$$
\tilde{x}_{k}^{(1)}=\frac{x_{k}^{(1)}}{\sqrt{\beta_{x}^{(1)}}}
$$

The (linear) action can then be estimated from

$$
\left(2 J_{x k}\right)^{*}=\left(\tilde{x}_{k}^{(1)}\right)^{2}+\left(\tilde{p}_{x k}^{(1)}\right)^{2}
$$

Similar results hold for the vertical plane.

A simulation is shown in Fig. 8. By using multiple pairs of BPMs the spatial distribution of the driving terms can be determined. Note, a PCA approach is not effective in this case, since the corresponding components are variance based rather than physics based. In other words, not directly related to the driving terms [29]. The estimator can be improved by including the effect of noise which leads to a Kalman filter [31-32]. Essentially, an optimal (recursive) solution of the generalized least-square problem ${ }^{1}$, i.e., for time-varying parameters.

1. Originally formulated by Gauss 1795 for fixed parameters and rediscovered by Legendre 1806. 


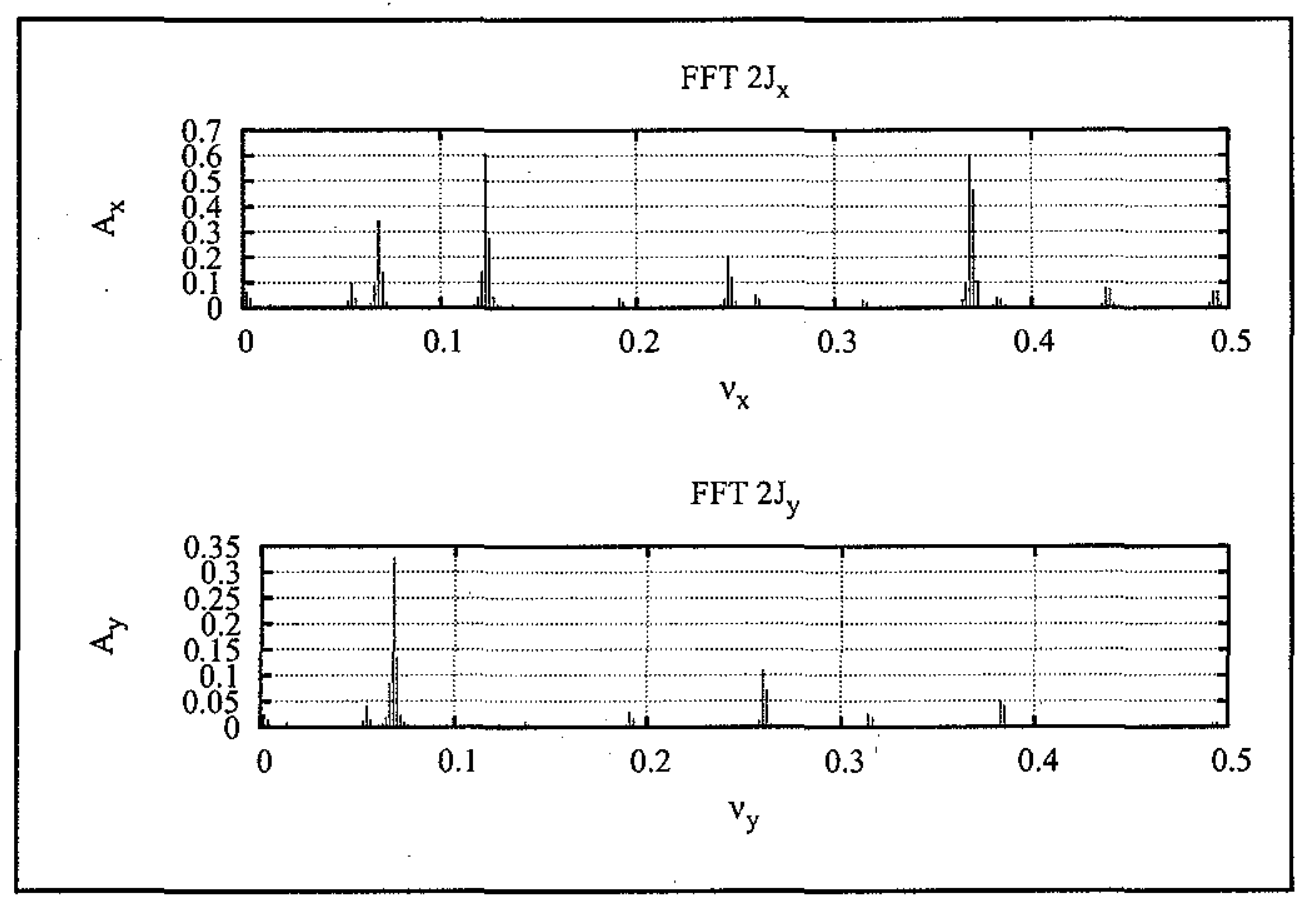

FIGURE 8. Spectrum of Reconstructed $2 J_{x, y}$. 


\subsection{An Optimal Predictor-Corrector Algorithm: the Kalman Filter}

The (linearized) dynamic (stochastic) process model is

$$
\bar{x}_{k}=A \bar{x}_{k-1}+\bar{w}_{k-1}
$$

with the (stochastic) measurement model

$$
\bar{z}_{k}=H \bar{x}_{k}+\bar{v}_{k},
$$

where $A$ is the (linear) one-turn map, and the random variables $\bar{w}_{k}$ and $\bar{v}_{k}$ represent the process- and measurement noise, respectively. They are assumed to be independent, normally distributed white noise

$$
p(w) \sim N(0, Q) \quad p(v) \sim N(0, R),
$$

with covariance matrices $Q$ and $R$.

The time update is given by (predictor)

$$
\begin{aligned}
& \ddot{x}_{k \mid k-1}=A \ddot{x}_{k-1}, \\
& P_{k \mid k-1}=A P_{k-1 \mid k-1} A^{T}+Q
\end{aligned}
$$

and the measurement update by (corrector)

$$
\begin{aligned}
S_{k} & =H P_{k \mid k-1} H^{T}+R, \\
K_{k} & =P_{k \mid k-1} H^{T} S_{k}^{-1}, \\
\hat{x}_{k} & =\hat{x}_{k \mid k-1}+K_{k}\left(\bar{z}_{k}-H \hat{x}_{k \mid k-1}\right), \\
P_{k \mid k} & =\left(1-K_{k} H\right) P_{k \mid k-1}
\end{aligned}
$$

where $P_{k \mid k-1}$ is the error covariance

$$
P_{k \mid k-1} \equiv \operatorname{cov}\left(\bar{x}_{k}-\hat{x}_{k-1}\right)=\mathrm{E}\left[\left(\bar{x}_{k}-\hat{x}_{k-1}\right)\left(\bar{x}_{k}-\hat{x}_{k-1}\right)^{T}\right]
$$

and similarly for $P_{k \mid k}$.

In particular, it is an (recursive) optimal solution for $K_{k}$ that minimizes the variance $P_{k \mid k}$ at each time step for the system ${ }^{1}$

1. Essentially a weighted average of the previous state, updated by the model, and the measurements. One may compare with classical navigation, i.e., dead reckoning and periodically a fix from the sextant. 


$$
\bar{x}_{k \mid k-1}=\left(1-K_{k}\right)\left(A \hat{x}_{k-1}+\bar{w}_{k-1}\right)+K_{k}\left(\bar{z}_{k}-\hat{z}_{k}\right),
$$

and correspondingly

$$
\begin{aligned}
P_{k \mid k} & =\left(1-K_{k}\right)\left(A P_{k-1 \mid k-1} A^{T}+Q\right)\left(1-K_{k}\right)^{T}+K_{k} R K_{k}^{T} \\
& =\left(1-K_{k}\right) P_{k \mid k-1}\left(1-K_{k}\right)^{T}+K_{k} R K_{k}^{T}
\end{aligned}
$$

which in this case simplifies to

$$
P_{k \mid k}=\left(1-K_{k} H\right) P_{k \mid k-1}
$$

In our case, the (linear) one-turn map is a rotation $A=R_{1 n}$ in Floquet space and $H=I$.

A simulation for the NSLS-II lattice with a BPM rms noise of 100 microns is shown in Figs. 9-10 for a betatron amplitude of $A_{x, y}=[1,1]$ and $A_{x, y}=[10,2] \mathrm{mm}$, respectively. The covariance is initially set to a large number so that most of the weight is on the measurements. In the first case, the filter is reducing the noise considerable by shifting the weight to the extra information from the "dead reckoning" by the (linear) model. In the latter, this is no longer the case due the model errors caused by the nonlinear effects at large amplitudes. Generalizing, we improve the filter by using a 7th order Taylor map for the model, see Fig. 11. While it works in this case, the approach is no longer self-consistent because the initially assumed normal distributions are not eigenmodes for the (nonlinear) system.

Clearly, the same (linear) technique could be used to improve the performance of e.g. the fast orbit correction feedback system, i.e., the (linears) system is now

$$
\begin{aligned}
\Delta \bar{x}(t) & =B \bar{u}(t)+\bar{w}(t), \\
\bar{z}(t) & =H \bar{x}(t)+\bar{v}(t)
\end{aligned}
$$

where $\bar{u}(t)$ are the kicks from the fast orbit correctors and $B$ the orbit response matrix. 


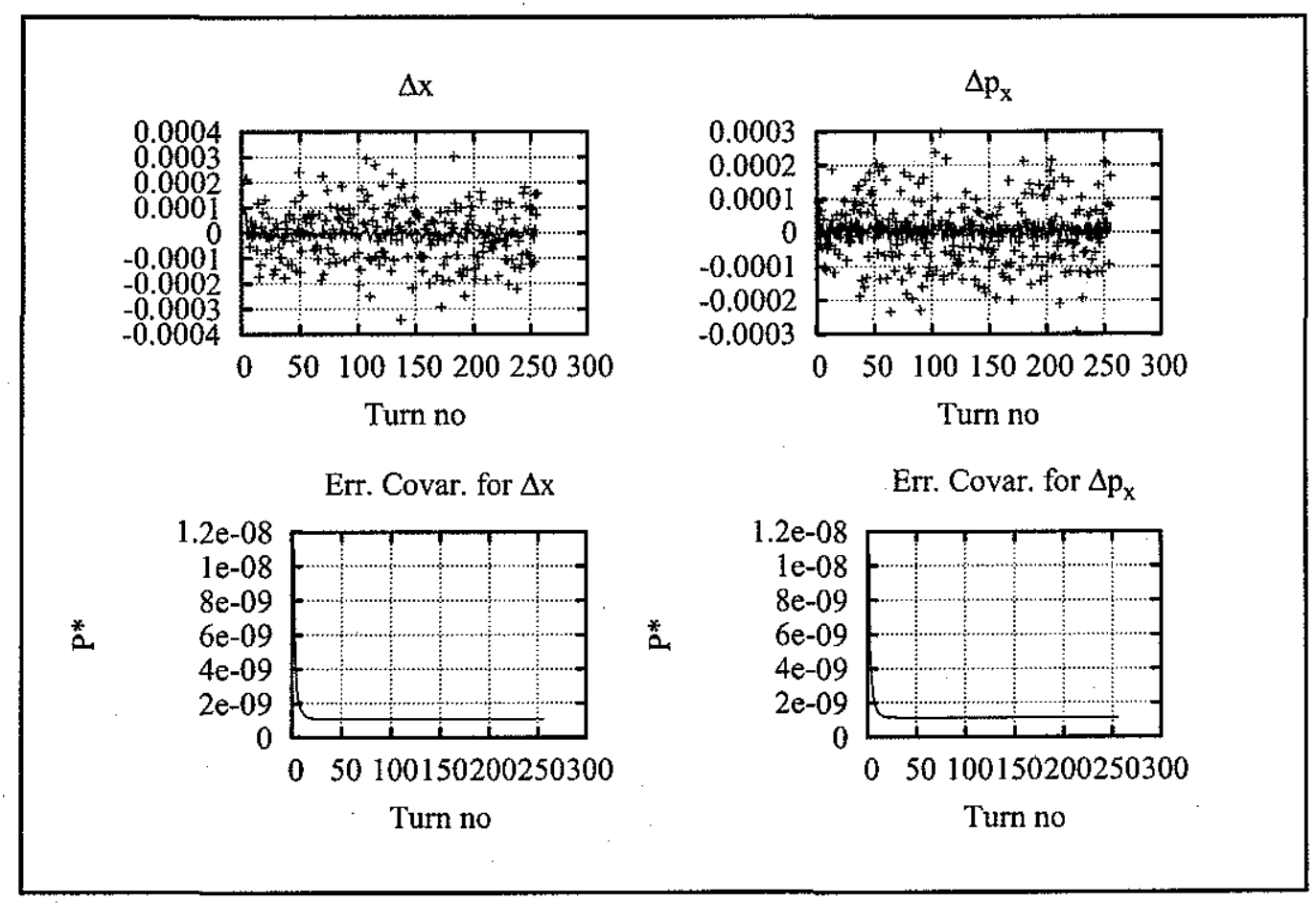

FIGURE 9. A State Estimator with Kalman Filter $\left(A_{x, y}=[1,1] \mathrm{mm}\right)$.

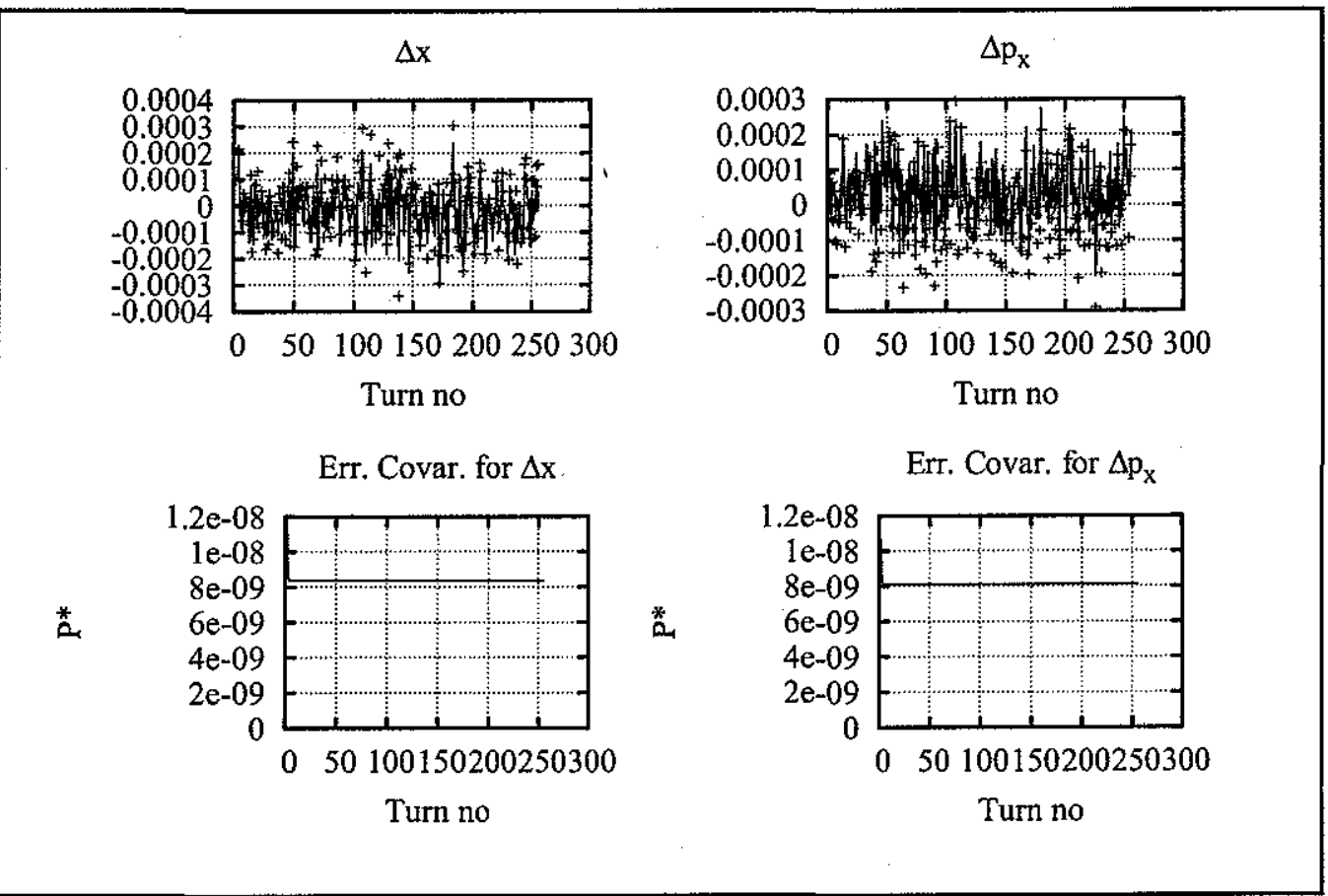

FIGURE 10. A State Estimator with Kalman Filter $\left(A_{x, y}=[10,2] \mathrm{mm}\right)$. 


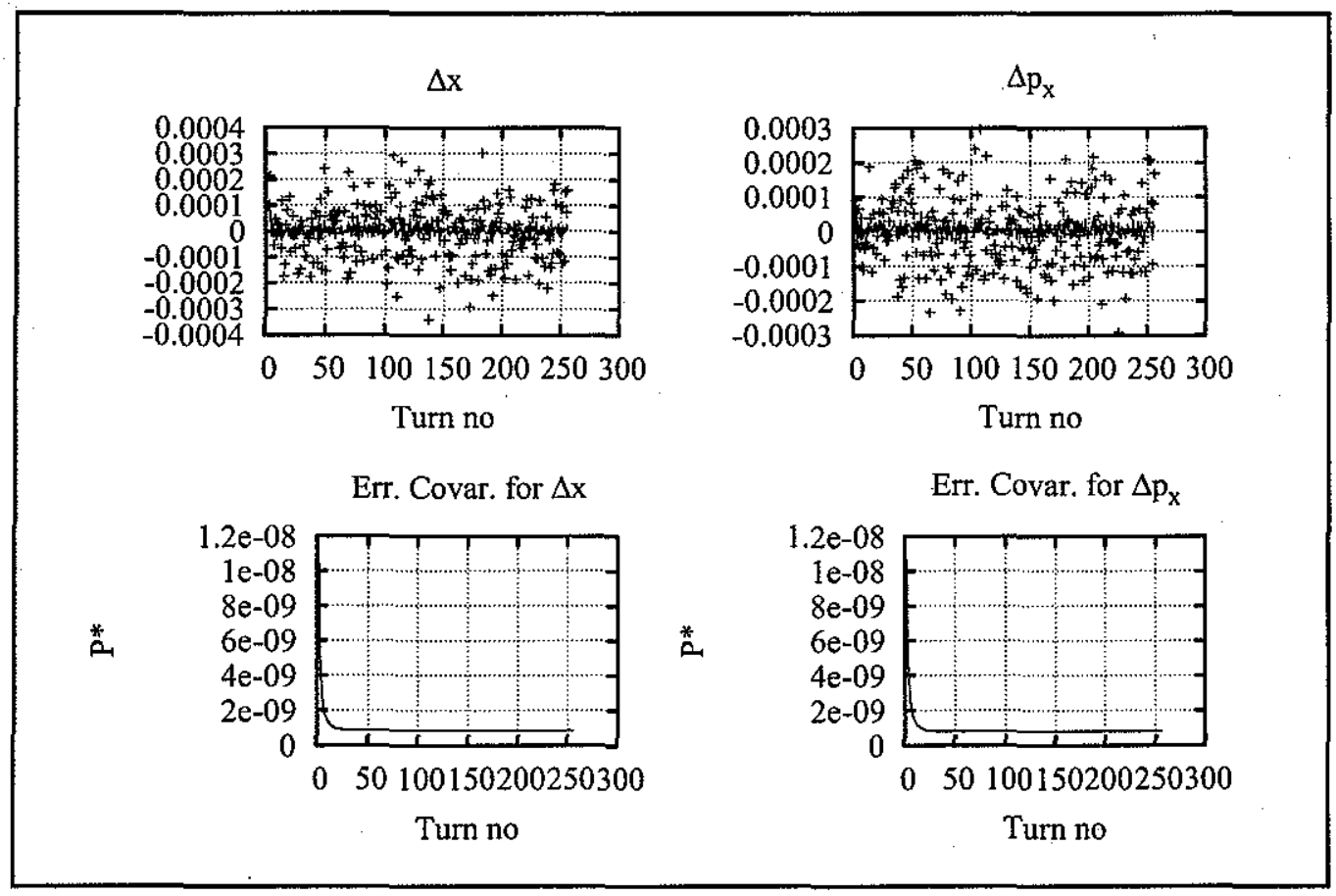

FIGURE 11. A State Estimator with Kalman Filter and 7th Order Taylor Map $\left(A_{x, y}=[10,2] \mathrm{mm}\right)$ 


\subsection{Requirements for NSLS-II Pinger}

Ideally, the basic requirements are:

- The kick should be shorter than one turn, i.e., $792 / 3 \times 10^{8}=2.6 \mu$ sec.

- It would kick the beam out to the physical aperture. However, since the injection amplitude is $\sim 10 \mathrm{~mm}$ in the horizontal plane and an (optimistic) estimate for the dynamic aperture is $\sim 15 \mathrm{~mm}, \beta_{x} \approx 20 \mathrm{~m}$, we will use the latter. The vertical plane is limited to $\pm 12.5 \mathrm{~mm}, \tilde{\beta}_{y} \approx 25 \mathrm{~m}$; due to the physical vertical aperture in the dipoles.

A horizontal kick $\theta_{x i}$ at a location $i$ generates the betatron motion

$$
x(s)=\theta_{x i} \sqrt{\beta_{x i} \beta_{x}(s)} \sin \left(\Delta \mu_{x i}(s)\right)
$$

and it follows that

$$
\theta_{x i}=\frac{\ddot{x}}{\sqrt{\beta_{x i} \beta_{x}}}
$$

And similarly for the vertical plane. The beta functions just downstream of the matching section to a long straight are:

$$
\beta_{x i} \approx 20 \mathrm{~m}, \quad \beta_{y i} \approx 8 \mathrm{~m}
$$

which gives

$$
\hat{\theta}_{x i}=\frac{15 \times 10^{-3}}{\sqrt{20 \times 20}}=0.8 \mathrm{mrad}, \quad \hat{\theta}_{y i}=\frac{12.5 \times 10^{-3}}{\sqrt{8 \times 25}}=0.9 \mathrm{mrad} .
$$




\subsection{Application to NSLS-II}

For the NSLS-II commissioning it is desirable to:

1. Measure the on- and off momentum dynamic aperture. This could be done with the injection kicker in the horizontal plane, but a pinger is required for the vertical. The required amplitude is $\pm[15,12.5] \mathrm{mm}$ at the center of the injection straight in the horizontal plane and the physical vertical aperture of the dipoles, respectively. Complementary, the dynamic aperture can also be measured with the scrapers.

2. Measure the tune footprint. The best (most precise approach) is to use a pinger for each plane; rather than the injection kicker for the horizontal. In particular, with a kick that is shorter than one turn, i.e., $2.6 \mu \mathrm{sec}$. If they are located just downstream of the matching section to a long straight, the beta functions are $[20,8] \mathrm{m}$, i.e., the required kicks are $[0.8,0.9]$ mrad.

There is a concept, known as e.g. TQM (Total Quality Management) in the U.S.; or simply quality control in northern Europe. In this country it is associated with the name W.

Edwards Deming [35]. However, he is best known for his work in Japan (in particular the car industry); which started 1950. Interestingly, he was decorated by the Japanese government 1960 and the U.S. President 1987. TQM is simply how to deliver (to the end user aka customer), timely and cost effectively, on a product with claimed performance. For example, one may compare the successful Apollo program with the organizations' later attempt for an inexpensive delivery system to put satellites, etc. into space. Or the successful technical culture fostered at the NACA Transonic Wind Tunnel; aka engineering-science [36]; a precursor to NASA.

The dynamic aperture and tune footprint are key characteristics of the performance of the lattice. So, by measuring these and comparing with the (nonlinear) model during commissioning, one can directly assess if the guidelines, requirements, etc. have been met by all the subsystems, e.g. the mechanical- and magnetic tolerances; i.e., if the entire system is performing as expected. And, in case of a gap between what's measured vs. predicted, at least one would have a diagnostic. In particular, an in-depth gap analysis can be pursued by:

3. Measure the betatron (or linear action variable) spectrum and compare with the model.

In fact, we have been using this technique to deconvolute issues with, and provide guidelines for: working points, magnet tolerances, insertion devices, etc. by analyzing data obtained from simulations with our computer model throughout the entire design process. Simply put: TQM.

On a trivial level, e.g. a polarity error in a sextupole would be apparent. But, more important, e.g. unacceptable higher order multipole errors in the magnets would have a unique signal in the spectrum. In fact, if all the BPMs have turn-by-turn capability (as planned), one can even obtain spatial information. An example is shown in Fig. 12 where we have introduced a systematic decapole component to one of the sextupole families. It generates 
a clear "signal" from $3 v_{x} \pm 2 v_{x}(0.24,0.018)$ (perturbed tunes). Analysis of its amplitudeand phase gives direct information about the source's integrated multipole strength- and location narrowed down to a set of discrete locations $(\bmod 2 \pi)$. If all the BPMs have turnby-turn capability, it can be further localized.

Similarly, when e.g. the damping wigglers are introduced into the lattice, one would have a direct diagnostic to validate that their field quality is satisfactory. And, if not, a method to determine why, by beam based measurements; rather than having to bring the device back to the lab (or manufacturer) for refined magnetic measurements. Similarly, the same technique can be applied for each insertion device that is introduced into the ring, i.e., ideally, integrated into a complex nonlinear system; without causing a show stopper. Lessons learnt (by one device) do exist: ALS (Apple-II EPU), SPEAR3 (wiggler), CHESS (wiggler).

With more advanced techniques one can also pursue on-line nonlinear corrections. For example, in the case of a major deficiency with: dynamic aperture, injection efficiency, or Touschek life time, e.g. at large positive chromaticities, specific nonlinear magnetic elements to control a particular resonance, identified from the spectrum, could potentially be retrofitted.

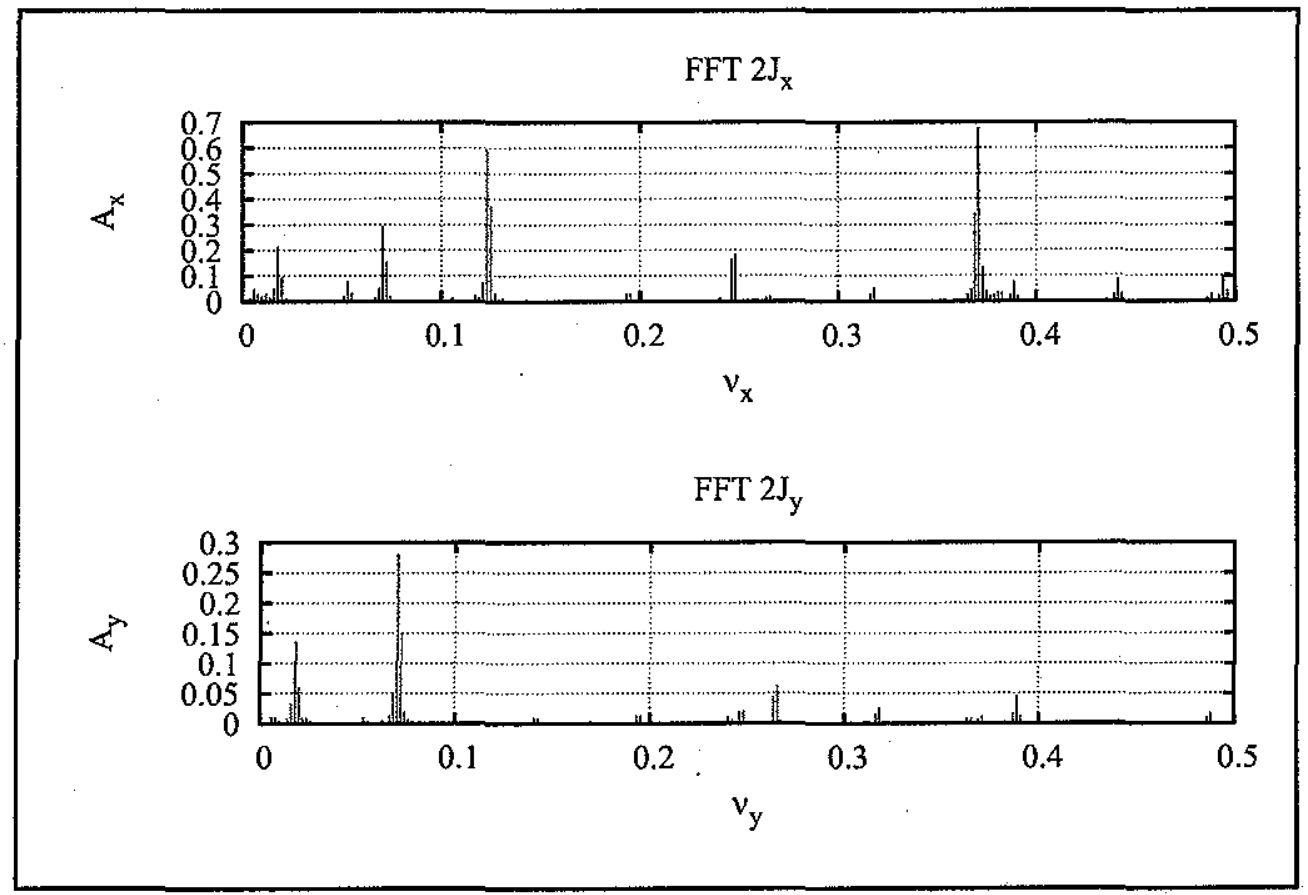

FIGURE 12. Spectrum of $2 J_{x, y}$ with a Decapole Component in the Lattice. 


\subsection{Conclusions}

To summarize:

- We have outlined how to make a basic calibration of the nonlinear model for synchrotrons. In particular, we have shown how this was done for LEAR, CERN (antiprotons) in the mid-80s [3]. Specifically, our accuracy for frequency estimation was $\sim 1 \times 10^{-5}$ for 1024 turns (to calibrate the linear optics) and $\sim 1 \times 10^{-4}$ for 256 turns for tune footprint and betatron spectrum. For a comparison, the estimated tune footprint for stable beam for NSLS-II is $\sim 0.1$. Since the transverse damping time is $\sim 20 \mathrm{msec}$, i.e., $\sim 4,000$ turns. There is no fundamental difference for: antiprotons, protons, and electrons in this case.

- Because the estimated accuracy for these methods in the field of particle accelerators has been naive, i.e., ignoring the impact of noise, we have also derived explicit formula, from first principles, for a quantitative statement. For e.g. $N=256$ and $5 \%$ noise we obtain $\delta v \sim 1 \times 10^{-5}$. A comparison with the state-of-the-arts in e.g. telecomm and electrical engineering since the $60 \mathrm{~s}$ is quite revealing. For example, Kalman filter (1960) [31-32], crucial for the: Ranger, Mariner, and Apollo (including the Lunar Module) missions during the 60s, see e.g. ref [37]. Or Claude Shannon et al since the 40s for that matter [12-13]. Conclusion: what's elementary in the latter is considered "advanced", if at all, in the former. It is little surprise then that published measurements typically contains neither error bars (for the random errors) nor estimates for the systematic in the former discipline.

- We have also showed how to estimate the state space by turn-by-turn data from two adjacent BPMs. And how to improve the resolution of the nonlinear resonance spectrum by Fourier analyzing the linear action variables instead of the betatron motion [29]. In fact, the state estimator could be further improved by adding a Kalman filter.

- For transparency, we have also summarized on how these techniques provide a framework- and method for a TQM (Total Quality Management) approach for the main ring.

Of course, to make the $(\$ 2.5 \mathrm{M})$ turn-by-turn data acquisition system that is being implemented (for all the BPMs) useful, a means ( $\sim 10 \%$ contingency for the BPM system) to drive the beam is obviously required.

\section{Acknowledgement}

I would like to thank I. Pinayev for an in-depth, intuitive brainstorming on the impact of noise on signal processing systems. And for having scrutinized Section 2.0. I would also like to thank S. Krinsky for having reviewed the entire document with care. In particular, his interest in, and analysis of, a SVD based approach for multiple BPM turn-by-turn data analysis triggered our curiosity to look into this approach further. 


\section{References}

[1] J. Bengtsson "Model Based Control of Nonlinear Dynamics" Lecture given to the Acc. Phys. Journal Club Apr 13, 2010.

[2] G. Portmann, J. Safranek “MATLAB Based LOCO”EPAC02, 1184-1186.

[3] J. Bengtsson "Non-Linear Transverse Dynamics for Storage Rings with Applications to the Low-Energy Antiproton Ring (LEAR) at CERN" CERN Yellow Report 88-10 (1988).

[4] P. Castro "Applications of the 1000-Turns Orbit measurement System at LEP" PAC99, 456-460.

[5] M. Bai, S. Peggs, T. Roser, T. Satogata, D. Trbojevic "Measuring Beta Function and Phase Advance with an AC Dipole" PAC03, 2204-2206.

[6] J. Bengtsson "NSLS-II: Control of Dynamic Aperture" BNL-81770-2008-IR (2008).

[7] J. Laskar, C. Froeschlé, A. Celletti "The Measure of Chaos by the Numerical Analysis of the Fundamental Frequencies. Application to the Standard Mapping" Physica D 67, 257-281 (1993).

[8] R. Bartolini, F. Schmidt "Tune Evaluation in Simulations and Experiments" CERN SL/95-84 (A) (1995).

[9] R. Bartolini, I. P. S. Martin, J. H. Rowland, P. Kuske, F. Schmidt "Correction of Multiple Nonlinear Resonances in Storage Rings" Phys. Rev. ST Accel. Beams 11, 104002 (2008).

[10] A. Streun, M. Böge, N. Abreu, M. Aiba, A. Lüdeke, § Andersson, J. Bengtsson, "Nonlinear Beam Dynamics Studies at the SLS" 2nd Workshop on Nonlinear Beam Dynamics in Storage Rings, DIAMOND, Nov 2-4, 2009.

[11] L. Yang, C. Steier "Experimental Frequency Map Analysis Using Multiple BPMs" PAC09.

[12] C. E. Shannon "A Mathematical Theory of Communication" Bell Syst. Tech. J. 27. 379-423, 623-656 (1948).

[13] C. E. Shannon "Communication in the Presence of Noise" Proc. Institute of Radio Engineers 37, 10-21(1949).

[14] P. Rudnick "Note on the Calculation of Fourier Series" Math. Comp. 20, 429-430 (1966).

[15] J. Binney, D. Spergel "Spectral Stellar Dynamics" Astrophys. J. 252, 308-321 (1982). 
[16] J. W. Cooley, J. W. Tukey "An Algorithm for the Machine Calculation of Complex Fourier Series" Math. Comp. 19, 297-301 (1965).

[17] J. W. Cooley, "How the FFT Gained Acceptance" IEEE Sig. Proc. Mag. 9, 10-13 (1992).

[18] G. C. Danielson, C. Lanszos, J. Franklin "Some Improvements in Practical Fourier Analysis and Their Application to X-Ray Scattering from Liquids" Franklin Inst. 233. $\underline{365-380,435-452(1942)}$.

[19] M. T. Heideman, D. H. Johnson, C. S. Burrus "Gauss and the History of the Fast Fourier Transform" IEEE ASSP Mag., 1, (4), 14-21 (1984).

[20] C. F. Gauss "Nachlass: Theoria Interpolationis Methodo Nova Tractata" Band 3, 265 327 (Königliche Gesellschaft der Wissenschaften, Göttingen, 1866).

[21] IEEE "Standard for Digitizing Waveform Recorders" IEEE Std. 1057-1994 (1994).

[22] R. Bartolini, A. Bazzani, M. Giovannozzi, W. Scandale "Tune Evaluation in Simulations and Experiments" CERN SL/95-84 (1995).

[23] E. Asséo, J. Bengtsson, M. Chanel "LEAR Beam Stability Improvements Using FFT Analysis" EPAC88, 541-543.

[24] W. H. Press, B. P. Flannery, W. A. Teukolsky, W. T. Vetterling "Numerical Recipes" (Cambridge, N.Y., 2007).

[25] K. Pearson. "On Lines and Planes of Closest Fit to Systems of Points is Space" Philosophical Magazine Series 6(2), 559m572 (1901).

[26] H. Hotelling "Analysis of a Complex of Statistical Variables into Principal Components" Journal of Educational Psychology 24, 417-441 \& $\underline{498-520}$ (1933).

[27] S. Krinsky priv. comm.

[28] P. Comon "Independent Component Analysis: a New Concept?" Signal Processing $\underline{36(3), 287-314(1994) .}$

[29] J. Bengtsson "The Sextupole Scheme for the Swiss Light Source (SLS): An Analytic Approach" SLS Note 9/97 (1997).

[30] J. Bengtsson, D. Briggs, G. Portmann "A Linear Control Theory Analysis of Transverse Coherent Bunch Instabilities Feedback Systems (A Control Theory Approach to Hill's Equation)" CBP Tech Note-026, PEP-II AP Note 28-93 (1993).

[31] R. E. Kalman "A New Approach to Linear Filtering and Prediction Problems" Trans. ASME, J. Basic Eng. 82D, 34-45 (1960). 
[32] R. E. Kalman, R. S. Bucy "New Results in Linear Filtering and Prediction Theory" Trans. ASME, J. Basic Eng. 83D, 95-108 (1961).

[33] C. F. Gauss "Theoria motus corporum coelestium: in sectionibus conicis solem ambientium" (Perthes \& Besser, Hamburqi, 1809).

[34] A. M. Legendre "Nouvelles méthodes pour la determation des orbites des comètes" (Didot, Paris, 1805).

[35] The W. Edwards Deming Institute.

[36] P. M. Back (editor) "From Engineering Science to Big Science" NASA SP-4219 (1998).

[37] W. S. Widnall "The Minimum-Time Thrust-Vector Control Law in the Apollo LunarModule Autopilot" Automatica 6(5), 661-672 (1970). 